\title{
Padronização de um protocolo específico para determinação da aptidão anaeróbia de nadadores utilizando células de carga
}

\author{
Marcelo Papoti ${ }^{1}$ \\ Luiz Martins ${ }^{2}$ \\ Sergio Cunha ${ }^{1}$ \\ Alessandro Zagatto ${ }^{1}$ \\ Claudio Gobatto ${ }^{1}$
}

\author{
${ }^{1}$ Laboratório de Biodinâmica UNESP Rio Claro \\ SP, Brasil. \\ ${ }^{2}$ Laboratório de Instrumentação para Fisiologia \\ do Exercício UNICAMP \\ SP, Brasil.
}

https://doi.org/10.5628/rpcd.03.03.36

\section{RESUMO}

O objetivo do presente estudo foi padronizar um protocolo específico para determinar a aptidão anaeróbia $\left(\mathrm{AP}_{\mathrm{ANA}}\right)$ de nadadores durante esforços de 30 segundos e suas correlações com as performances de $200 \mathrm{~m}\left(\mathrm{P}_{\mathrm{MÁX}} 200\right)$ e $400 \mathrm{~m}\left(\mathrm{P}_{\mathrm{MÁX}} 400\right)$ nado crawl. Para avaliar a $\mathrm{AP}_{\mathrm{ANA}}$, assumida como o impulso em nado atado $\left(\mathrm{IMP}_{\mathrm{NA}}\right)$, foi desenvolvido um ergômetro tendo células de carga como elemento sensor primário, conectadas ao nadador por um fio preso em suas cinturas. O teste consistiu na realização de 2 esforços máximos $\left(\mathrm{IMP}_{\mathrm{NA}}-1\right.$ e $\left.\mathrm{IMP}_{\mathrm{NA}}-2\right)$ com duração de 30 s com amostras sanguíneas $(25 \mu \mathrm{l})$ coletadas ao final de cada esforço para determinação da lactacidemia $\left([\mathrm{lac}]_{\text {pico }}\right)$. Para as possíveis diferenças entre os valores de IMP $_{N A}$, e sua correlação com as $\mathrm{P}_{\mathrm{MÁx}} 200$ e $\mathrm{P}_{\mathrm{MÁx}} 400$, foram utilizados o teste-t pareado e análise de correlação de Pearson respectivamente, com nível de significância pré-fixado para $p<0,05$. Não houve diferenças significativas entre os valores da $[\mathrm{lac}]_{\text {pico-1 }}(6,8 \pm 03 \mathrm{mmol} / \mathrm{L})$ e $[\mathrm{lac}]_{\text {pico-2 }}(6,5 \pm 0,4 \mathrm{mmol} / \mathrm{L})$ e entre o $\operatorname{IMP}_{\mathrm{NA}-1}\left(2510,2 \pm 105\right.$ N.s) e $\operatorname{IMP}_{\mathrm{NA}-2}(2541,9 \pm 115,2$ N.s $)$. Foi observada significativa correlação entre os valores de $\mathrm{IMP}_{\mathrm{NA}}-1 \mathrm{e}$ $\mathrm{IMP}_{\mathrm{NA}}-2(\mathrm{r}=0,93)$. Além disso, esses valores foram significativamente correlacionados com as $\mathrm{P}_{\text {MÁX }} 200(0,86)$ e $\mathrm{P}_{\mathrm{MÁX}} 400$ $(0,63)$. De posse dos resultados, pode-se concluir que o $\operatorname{IMP}_{\mathrm{NA}}$ representa a $\mathrm{AP}_{\mathrm{ANA}}$ e pode ser utilizado como preditor de performance em provas de 200 e $400 \mathrm{~m}$ nado crawl.

Palavras-chave: natação, aptidão anaeróbia, nado atado, lactato, performance.

\begin{abstract}
Standardization of a specific protocol to determine the anaerobic conditioning in swimmers during a 30sec effort using load cells

The purpose of this study was to standardize a specific protocol to determine the anaerobic conditioning $\left(A C_{A N A}\right)$ in swimmers during a 30 sec effort and its correlation with performances of $200 \mathrm{~m}\left(P_{\text {MAX200 }}\right)$ and $400 \mathrm{~m}\left(P_{\text {MAX400 }}\right)$ crawl style. $A C_{A N A}$, regarded as the impulse of tethered swimming $\left(I M P_{N A}\right)$, was assessed with an ergometer having load cells as primary sensing element. They were connected to the swimmers by an attached wire at their waist. Two maximal efforts $\left(I M P_{N A}-1\right.$ and $\left.I M P_{N A}-2\right)$ were performed with 30 sec of duration. Blood samples were taken at the end of each effort to determine lactatemy ([lac]peak). Paired t test and Pearson's correlation were used with significance level of $p<0.05$. No differences were observed between the values of [lac]peak-1 $(6.8 \pm 03 \mathrm{mmol} / \mathrm{L})$ and [lac]peak-2 $(6.5 \pm 0.4 \mathrm{mmol} / \mathrm{L})$ and between the $\mathrm{IMP}_{\mathrm{NA}^{-}}-1(2510.2 \pm 105.0 \mathrm{N.s})$ and $I M P_{N A}-2(2541.9 \pm 115.2$ N.s). A significant correlation was observed between the values of $I M P_{N A}-1$ e IMP ${ }_{N A}-2(r=0.93)$. In addition, these values were correlated significantly with the $P_{\text {MAX200 }}$ $(r=0.86)$ and $P_{M A X 400}(r=0.63)$. Based on these data it can be concluded that the $I M P_{N A}$ represents the $A C_{A N A}$ and it may be used as predictor of performances in tests of 200 and $400 \mathrm{~m}$ swim crawl.
\end{abstract}

Key Words: swimming, anaerobic conditioning, swim tethered, lactate, performance. 


\section{INTRODUÇÃO}

Os testes que avaliam a aptidão anaeróbia não estão tão bem desenvolvidos como os que avaliam as qualidades aeróbias, embora variáveis anaeróbias como força e potência sejam aspectos importantes para a evolução do nadador (24). Maglischo (18) sugeriu como forma de avaliar a capacidade anaeróbia a determinação da concentração de lactato sanguíneo após esforços máximos, sendo que baixos valores de lactato, juntamente com desempenhos insatisfatórios, poderiam indicar a deterioração desta capacidade. O Teste de Wingate proposto por Bar-Or et al. (2) é muito utilizado na avaliação da potência anaeróbia de membros inferiores (3) e superiores (6), pois é um teste reprodutível $(3,28)$ e apresenta significativas correlações com índices anaeróbios, como a máxima concentração de lactato e o déficit de oxigênio (3). Hawley \& Williams (10) encontraram elevada correlação $(r=0,83)$ entre o Teste de Wingate em ergômetro de braço e a velocidade de $50 \mathrm{~m}$ nado crawl. Em contradição, Guglielmo \& Denadai (9) não verificaram qualquer correlação entre as velocidades de $14 \mathrm{~m}, 25 \mathrm{~m}, 50 \mathrm{~m}$ e $400 \mathrm{~m}$ nado crawl e os parâmetros obtidos após o Teste de Wingate utilizando um ergômetro de braço isocinético (concentração de lactato, índice de decaímento e potências pico e média) e concluíram que essa metodologia não fornece indicativos da aptidão anaeróbia (lática e alática) de nadadores. Sharp et al. (23) constataram correlações de $r=0,90$ entre a velocidade máxima de 25 jardas no nado crawl e a potência de braçada em 40 nadadores utilizando um banco de nado ("swim bench"), descrito como um dispositivo de resistência, composto por 2 cabos com palmares ("handpaddles") em uma de suas extremidades, permitindo a simulação da braçada do nado fora da água e a regulação da velocidade na proporção que uma determinada força é aplicada. Swaine \& Zanker (26) concluíram que o "swim bench" é um ergômetro reprodutível na avaliação do consumo máximo de oxigênio $(r=0,98)$ e da freqüência cardíaca $(0,88)$ de nadadores altamente treinados, possibilitando o monitoramento dos efeitos do treinamento. Os autores ainda especularam que esse ergômetro deva também ser confiável para estudos envolvendo respostas bioquímicas no nado simulado, como o acúmulo de lactato no sangue. No entanto, o grupo de nadadores investigado por Sharp et al. (23) apresentou grandes variações na velocidade de nado, podendo favorecer a obtenção de correlações elevadas. Além disso, os melhores nadadores não necessariamente produzem os valores mais elevados de potência no "swim bench" (15), evidenciando assim a necessidade de ergômetros mais específicos para a determinação da força e potência de nado. Costill et al. (7) adaptaram um sistema "biocinético" para mensurar a força e potência específica na piscina com nado parcialmente-atado em 76 nadadores universitários.

Constataram elevada correlação $(0,84)$ entre potência e velocidade máxima de nado em distâncias curtas e concluíram que este sistema fornece um método sensível de avaliação da potência específica para nadadores. D`Acquisto \& Costill (8) encontraram correlações de $0,64(p=0,11)$ e $0,87(p<0,05)$ entre a potência obtida através de nado parcialmente atado e a velocidade ("sprint") de nado peito para homens e mulheres, respectivamente. Além disso, relataram significativas correlações de 0,91 e 0,86 , para as performances de $91,4 \mathrm{~m}$ e $365,8 \mathrm{~m}$ em nado peito, respectivamente $(\mathrm{p}<0,05)$, demonstrando que a habilidade de gerar potência durante o nado parcialmente atado foi o preditor de ambas performances de "sprint" e endurance. Também são freqüentes a utilização de ergômetros que avaliam a força dos nadadores em nado totalmente atado $(13,19,22)$, por apresentarem elevada reprodutibilidade $(11,13)$ e correlações com as velocidades de nado nas distâncias entre $25 \mathrm{~m}$ e $200 \mathrm{~m}(19,22)$. Além disso, são sensíveis aos efeitos do treinamento $(14,21,22$, 27). No entanto, a maioria desses estudos submeteu os nadadores a esforços com duração de aproximadamente 10 segundos ou 20 braçadas, limitando assim a participação do sistema glicolítico. Desse modo, o objetivo do presente estudo foi verificar a reprodutibilidade de um protocolo específico para avaliar a aptidão anaeróbia de nadadores e suas correlações com as performances de $200 \mathrm{~m}$ e $400 \mathrm{~m}$ nado crawl.

\section{MATERIAIS E MÉTODOS \\ Participantes}

Participaram voluntariamente desse estudo, após manifestação por escrito do termo de consentimento aprovado pelo comitê de ética da UNESP campus de Rio Claro, 13 nadadores de 2 equipes de natação de 
Bauru (Associação Luso Brasileira de Bauru e Unimed SESI Prata), filiados a Federação Aquática Paulista, de nível estadual e nacional, com idade variando entre 15 e 18 anos e tempo mínimo de natação competitiva de 2 anos.

\section{Equipamento}

Foi desenvolvido um equipamento especialmente para determinar a força e o impulso em nado atado. Para isso, foi utilizado um dinamômetro com capacidade de $300 \mathrm{~N}$, contendo células de carga (strain gages) como elemento sensor primário a partir da aplicação elétrica de pontes de Weatstone (1/2 Bridge). O dinamômetro foi suspenso por um arranjo físico de madeira fixado ao solo e próximo à borda da piscina. No centro do dinamômetro foi conectado um fio de aço com $408 \mathrm{~cm}$ de comprimento que teve em sua extremidade oposta um cinto de nylon, preso à cintura do nadador, localizado a uma distância de $300 \mathrm{~cm}$ em relação à borda da piscina (Figura 1).

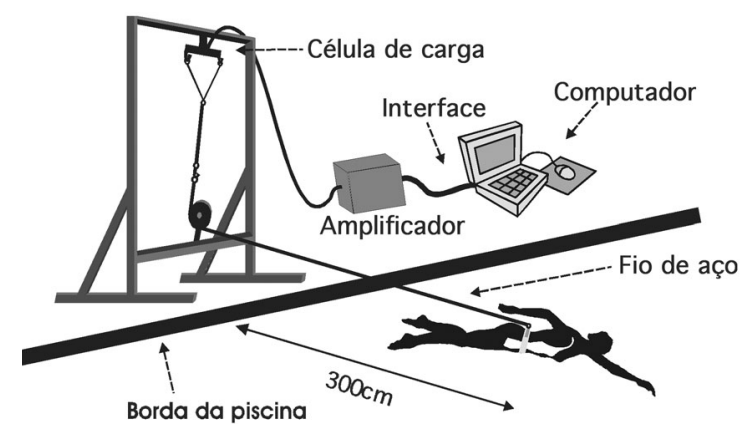

Figura 1. Modelo esquemático do sistema desenvolvido para determinar a força e impulso em nado atado.

O equipamento foi calibrado com a sobreposição de pesos, com incremento de aproximadamente 97 gramas. No dia do teste ainda foram utilizados como valores de referência pesos de $2 \mathrm{Kg}$ e $10 \mathrm{Kg}$.

\section{Testes}

O teste propriamente dito consistiu da aplicação de 2 esforços máximos em estilo crawl com duração de 30s e incentivo verbal dos atletas e pesquisadores e com intervalo passivo de aproximadamente 20 minutos, estando os nadadores amarrados ao aparato de medição. O início e o término do teste foram determinados por sinal sonoro (apito) após aproximadamente 10 s de nado moderado. Ao final dos esforços, foram coletadas amostras de sangue $(25 \mu \mathrm{l})$ do lóbulo da orelha nos minutos 1,3 e 5, para determinação da concentração pico de lactato ([lac $]_{\text {pico }}$ ). A deformação detectada pelas células de cargas (strain gage), devido à tensão gerada pelos esforços do nadador, foi amplificada por uma fonte de extensometria portátil (SODMEX ME-01D). Os valores obtidos durante os esforços foram enviados por uma interface ao computador e armazenados no programa para aquisição de dados Lab View na freqüência de $400 \mathrm{~Hz}$. Estes valores foram depois submetidos ao processo de análise residual e suavizados utilizando o filtro "butterworth" de quarta ordem com freqüência de corte de $3 \mathrm{~Hz}$. Com a utilização da reta de calibração, os valores obtidos foram convertidos em unidades de força $(\mathrm{N})$ pelo programa Matlab 5.3, possibilitando a determinação da força média $\left(\mathrm{FM}_{\mathrm{NA}}\right)$ e impulso em nado atado $\left(\mathrm{IMP}_{\mathrm{NA}}\right)$, obtido pela integral da força e o tempo de esforço, que foi assumido como índice de aptidão anaeróbia $\left(\mathrm{AP}_{\mathrm{ANA}}\right)$. Observou-se que os valores iniciais (aproximadamente 1s) foram bastante elevados, provavelmente devido aos esforços realizados durante a transição do nado moderado para o nado intenso (Figura 2).

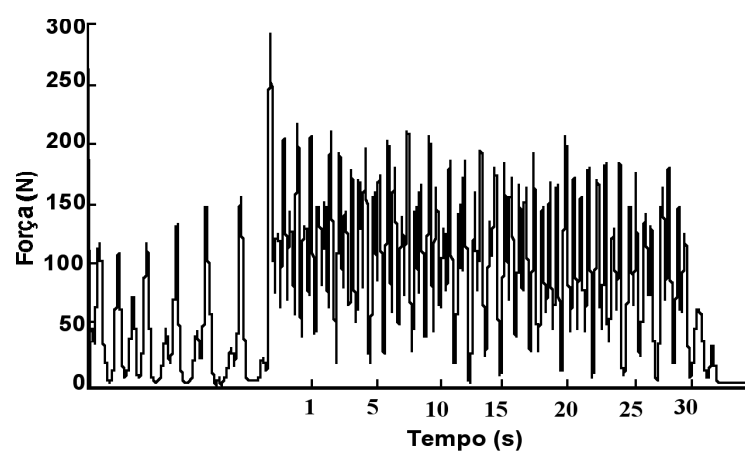

Figura 2. Exemplo do comportamento da força $(N)$ determinada em nado atado de um único atleta durante aproximadamente 10 s de nado moderado e esforço máximo durante 30 segundos. 
Desse modo, tal como nos procedimentos utilizados por TRAPPE et al. (27), os 400 pontos iniciais foram desconsiderados para não superestimar os valores reais (Figura 3).

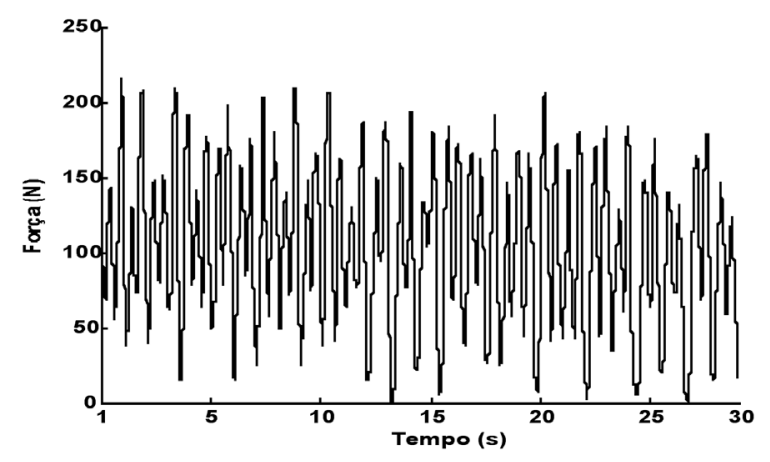

Figura 3. Comportamento da força determinada em nado atado ( $N$ ) de um único atleta, após descontados os 400 pontos iniciais do esforço máximo de 30 segundos.

Visando estabelecer a correlação entre a aptidão anaeróbia e a performance, foram realizados, após um período de 24 horas, esforços máximos (Pmáx) nas distâncias de $200 \mathrm{~m}\left(\mathrm{P}_{\text {máx }} 200\right)$ e $400 \mathrm{~m}\left(\mathrm{P}_{\text {máx }} 400\right)$ em estilo crawl, separados por um intervalo mínimo de 3 horas.

\section{Tratamento estatístico}

A $\mathrm{FM}_{\mathrm{NA}}, \mathrm{IMP}_{\mathrm{NA}}$ e $[\mathrm{lac}]_{\text {pico }}$ obtidas após esforços máximos de 30s, assim como as performances máximas de $200 \mathrm{~m}$ e $400 \mathrm{~m}$ em estilo crawl estão apresentadas em média \pm erro padrão da média. Os valores $\mathrm{FM}_{\mathrm{NA}}, \mathrm{IMP}_{\mathrm{NA}}$ e $[\mathrm{lac}]_{\text {pico, }}$ determinados no teste e reteste foram analisados pelo teste-t de student para amostras dependentes.

Com o objetivo de evitar redundância, somente os valores de $\mathrm{IMP}_{\mathrm{NA}}$ foram relacionados com a Pmáx (200m e 400m) a partir da análise de correlação de Pearson. Em todos os casos, o nível de significância foi pré-fixado para $\mathrm{p}<0.05$.

\section{RESULTADOS}

Os valores de $\mathrm{FM}_{\mathrm{NA}}, \mathrm{IMP}_{\mathrm{NA}}$ e $[\mathrm{lac}]_{\text {pico }}$ obtidos durante os 2 esforços de 30s em nado atado não apresen- taram diferenças significativas (Tabela 1). Além disso, os valores de $\operatorname{IMP}_{\mathrm{NA}}\left(\mathrm{IMP}_{\mathrm{NA}^{-}}-1\right.$ e IMP $\left.\mathrm{NA}^{-2}\right)$ foram significativamente correlacionados (Figura 4).

Tabela 1. Valores de força média $\left[F M_{N A}\right]$, impulso $\left[I M P_{N A}\right]$ e concentração pico de lactato ([lac $]_{\text {pico }}$ ), obtidos após 2 esforços máximos de 30 segundos em nado atado.

\begin{tabular}{lccc} 
& $\mathrm{FM}_{\mathrm{NA}}(\mathrm{N})$ & $\mathrm{IMP}_{\mathrm{NA}}(\mathrm{N} . \mathrm{s})$ & {$[\mathrm{lac}]_{\text {pico }}(\mathrm{mmo} / \mathrm{L})$} \\
\hline Esforço-1 & $86,6 \pm 3,6$ & $2510,2 \pm 105,0$ & $6,8 \pm 0,3$ \\
Esforço-2 & $87,6 \pm 4,0$ & $2541,9 \pm 115,2$ & $6,5 \pm 0,4$
\end{tabular}

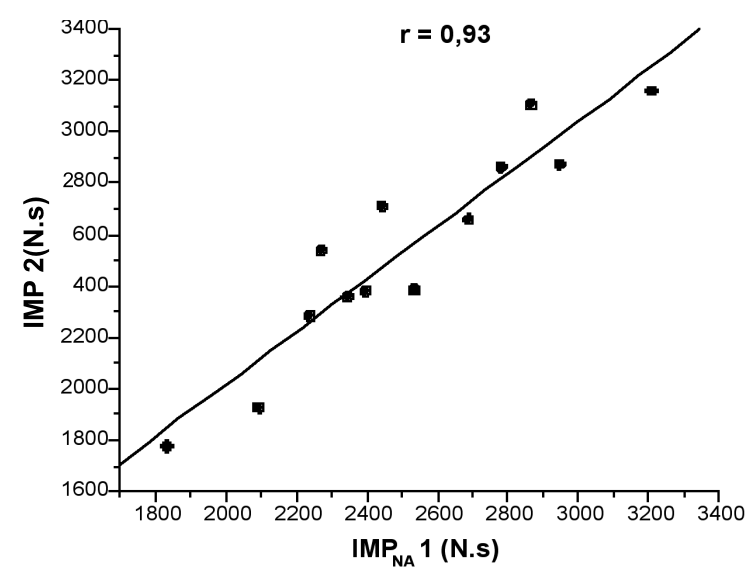

Figura 4. Correlação entre os valores de impulso em nado atado $\left(I M P_{N A}-1\right.$ e $\left.I M P_{N A}-2\right)$ obtidos após 2 esforços máximos de 30 segundos.

Também foram observadas elevadas correlações entre os valores de $\mathrm{IMP}_{\mathrm{NA}}$ e as Pmáx ${ }_{200}$ e Pmáx ${ }_{400}$ nado crawl (Tabela 2).

Tabela 2. Valores de dispersão ( $\mathrm{r}$ ] obtidos pela análise de correlação de Pearson.

\begin{tabular}{|c|c|c|c|}
\hline $\begin{array}{l}\mathrm{MP}_{\text {NA-1 VS }} \\
\mathrm{P}_{\text {mix }} 200\end{array}$ & $\begin{array}{l}\mathrm{MP}_{\mathrm{NA}-2 \mathrm{~V}} \\
\mathrm{P}_{\text {MXX }} 200\end{array}$ & $\begin{array}{l}\mathrm{MP}_{\mathrm{NA}-11 \mathrm{VS}} \\
\mathrm{P}_{\mathrm{Aix}} 400\end{array}$ & $\begin{array}{l}\text { IMP }_{\text {NA-Z Vs }} \\
P_{\text {Mí }} 400\end{array}$ \\
\hline $0,86^{*}$ & $0,84^{*}$ & $0,63^{*}$ & $0,54^{*}$ \\
\hline
\end{tabular}

*Correlação significativa para o nível prefixado $(p<0,05)$. 


\section{DISCUSSÃO}

No presente estudo os valores de teste e reteste apresentaram correlações de $r=0,92$, semelhante aos verificados em ergômetros que avaliam variáveis como força $(1 \mathrm{r}=0,72$ a 0,95$)$ e potência anaeróbia de membros inferiores $(3 \mathrm{r}=0,94)$ e em dinamômetros adaptados para realizar medidas em situação de nado atado utilizando células de carga como elemento sensor primário $(11 \mathrm{r}=0,91,13 \mathrm{r}=0,88$ a 0,95 , $19 \mathrm{r}=0,92)$. Além disso, os valores obtidos de $\mathrm{IMP}_{\mathrm{NA}}$ assim como os de lactacidemia, não foram significativamente diferentes entre as tentativas, o que confirma a elevada reprodutibilidade do equipamento desenvolvido, assim como o protocolo utilizado. Embora a mecânica do nado sofra alterações, principalmente pela diminuição da amplitude das braçadas $(16,17)$, os esforços em nado atado ou parcialmente atados são desenvolvidos em ergômetros específicos que permitem a mensuração de parâmetros fisiológicos, como por exemplo o $\mathrm{VO} 2_{\text {máx }}$, com a mesma fidedignidade quando mensurados em swimming flume e em nado livre (5). Além disso, os estudos que determinaram a força e potência em nado atado, através da realização de esforços anaeróbios, apresentaram elevadas correlações com a performance em provas rápidas $(7,19)$. No entanto, como foi dito anteriormente, a maioria desses estudos utilizaram esforços com duração de aproximadamente 10 segundos, limitando assim a participação do sistema glicolítico. Nesse sentido, foram utilizados no presente trabalho esforços com duração de 30 segundos, visando promover uma situação semelhante ao Teste de Wingate. Bar-Or (3) em revisão de literatura observou que os atletas de esportes com predominância anaeróbia (levantamento de peso, ginastas e lutadores) apresentavam valores de potência pico e média obtida em Teste de Wingate, superiores aos atletas praticantes de atividades consideradas aeróbias (corredores de $10 \mathrm{Km}$ e ultra-maratonistas). Jacobs et al. (12) constataram significativas reduções nas concentrações de adenosina trifosfato, creatina fosfato e glicogênio, seguido por um aumento de aproximadamente $51,5 \mathrm{mmol} . \mathrm{Kg}^{-1}$ na concentração de lactato muscular, em nove mulheres, após a realização de 30s de esforço máximo em cicloergômetro (Wingate). Striet et al. (25) relataram que o sistema fosfagênio fornece $24 \%$ a $27 \%$ da energia total con- sumida durante esforço máximo de 30s. Beneke et al. (4), verificaram que a energia fornecida durante a realização do Teste de Wingate é proveniente em aproximadamente $18,6 \%, 31,1 \%$ e $50,3 \%$ dos metabolismos aeróbio, anaeróbio alático e lático, respectivamente. Além disso, estudos utilizando o nado atado, com o mesmo tempo de duração, demonstraram significativa correlação $(p<0,05)$ entre a potência anaeróbia e a atividade neuromuscular (13) e com a performance de $200 \mathrm{~m}$ antes e após o período de polimento (22).

Os valores máximos de lactato no presente estudo foram de aproximadamente $6,5 \mathrm{mmol} / \mathrm{L}$. Guglielmo \& Denadai (9) encontraram valores de 7,78 $\pm 1,92$ $\mathrm{mmol} / \mathrm{L}$ após esforços máximos de 30s em ergômetro de braço isocinético. Weinstem et al. (28) ao investigarem a reprodutibilidade da concentração pico de lactato, freqüência cardíaca e volume plasmático após Teste de Wingate em membros inferiores, verificaram valores de aproximadamente $9,7 \mathrm{mmol} / \mathrm{L}$ de lactato, superiores aos obtidos em nado atado e ergômetro de braço. Uma possível explicação para a diferença encontrada nos valores de lactacidemia é a grande massa muscular envolvida durante esforços utilizando membros inferiores.

$\mathrm{O} \mathrm{IMP}_{\mathrm{NA}}$ nesse estudo foi assumido como um índice que fornece indiretamente a aptidão anaeróbia dos nadadores. Embora esse índice seja determinado de maneira não invasiva e necessite do empenho dos nadadores durante sua realização, os elevados valores de lactato sanguíneo, juntamente com as significativas correlações com as performances de $400 \mathrm{~m}$ e principalmente de $200 \mathrm{~m}$ nado crawl, evidenciam a significativa participação do sistema glicolítico durante a realização dos esforços. No entanto, a presente investigação não correlacionou os valores $\mathrm{IMP}_{\mathrm{NA}}$ com as performances de $50 \mathrm{~m}$ e $100 \mathrm{~m}$ nado crawl, que são distâncias oficiais de competição com predomínio do sistema anaeróbio lático (18). A maior correlação observada entre o $\mathrm{IMP}_{\mathrm{NA}}$ e a performance de $200 \mathrm{~m}$ em comparação com a performance de $400 \mathrm{~m}$ nado crawl, possibilita especular sobre a existência de uma relação inversa entre o $\mathrm{IMP}_{\mathrm{NA}}$ e a distância de provas oficiais de natação, à medida que aumenta a predominância do sistema glicolítico, embora mais pesquisas sejam necessárias para verificar essa hipótese. Desse modo, os resultados do pre- 
sente estudo sugerem que o $\operatorname{IMP}_{\mathrm{NA}}$ determinado durante esforços máximos de 30s utilizando células de carga é um protocolo reprodutível que possivelmente reflete a aptidão anaeróbia dos nadadores e pode ser utilizado como preditor de performance em provas de $400 \mathrm{~m}$ e $200 \mathrm{~m}$ nado crawl.

\section{CONCLUSÃO}

O impulso em nado atado, determinado com a realização de esforço máximo de 30 segundos utilizando células de carga, apresentou-se como uma ferramenta efetiva na predição de performance nas provas de $400 \mathrm{~m}$ e $200 \mathrm{~m}$ nado crawl.

\section{Nota dos autores}

Os autores agradecem o apoio da Fundação de Amparo à Pesquisa do Estado de São Paulo FAPESP na realização deste trabalho.

(Processo 01/08295-2).
CORRESPONDÊNCIA

\section{Marcelo Papoti}

Laboratório de Biodinâmica UNESP Rio Claro - SP. Av. 24 A 15-15, Bela Vista, 13506-900 Rio Claro-SP, Brasil. papoti@ig.com.br 


\section{REFERÊNCIAS BILIBOGRÁFICAS}

1 Avis F J, Hoving A, Toussaint HM (1985). A dynamometer for the measurement of force, velocity, work and power during an explosive leg extension. Eur J Appl Physiol 54:210-215.

2 Bar-Or O, Dotan R, Inbar O (1977). A 30-second all-out ergometric test its realiability and validity for anaerobic capacity. Israel Journal Medical Sciences 13:326.

3 Bar-Or O (1987). The Wingate Anaerobic Test: An Update on Methodology, Reliability and Validity. Sports Medicine 4:381-394.

4 Beneke R, Pollmann C, Bleif I, Leithauser HM (2002). How anaerobic is the Wingate Anaerobic Test for humans?. Eur J Appl Physiol 87:388-392.

5 Bonen A, Wilson BA, Yarkony M, Belcastro AN (1980). Maximal oxigen uptake during free, tethered, and flume swimming. J Appl Physiol 48:232-235.

6 Cameron JRB, Roache R, Hay JT, Bar-Or O (1988). Anaerobic power of arms in teenage boys and girls: relationship to lean tissue. Eur J Appl Physiol. 57:677-683.

7 Costill LD, Reifield F, Kirwan J, Thomas, R (1986). A computer based system for the measurement of force and power furing front crawl swimming. J Swimming Res 2:1619.

8 D`Acquisto LJ, Costill, DL (1998). Relationship between intracyclic linear body velocity flutuations, power and sprint breatstroke performance. J Swimming Res 13:8-14.

9 Gugielmo LGA, Denadai BS (2000). Assesment of Anaerobic Power of Swimmers: The Correlation of Laboratory Tests on an Arm Ergometer with Field Tests in a Swimming Pool. J Strength Cond Res 14:395-398.

10 Hawley JA, Williams MM (1991). Relationship between uper body anaerobic power and freestyle swimming performance. Int J Sports Med 12:1-5.

11 Hooper SL, Mackinnon LT, Ginn EM (1998). Effects of three tapering techniques on the performances, forces and psychometric measures of competitive swimmers. Eur J Appl Physiol 78:258-263.

12 Jacobs I, Bar-Or O, Karlsson J, Dotan R, Tesch P, Kaiser P, Inbar O (1982). Changes in muscle metabolites in females with 30-s exhaustive exercise. Med Sci Sports Exerc 14: 457460

13 John RS, Koceja DM, Stanger JM, Harms CA (1996). Mood neuromuscular function, and performance during training in female swimers. Med Sci Sports Exerc 28:372-377.

14 Johns RA, Houmard AJ, Kobe WR, Hortobágy T, Bruno JN, Wells MJ, Shinebarger HM (1992). Effects of taper on swim power, stroke distance, and performance. Med Sci Sports Exerc. 24:1141-1146.

15 Keskinen KL (1994). Measurement of technique in front crawl swimming. Med Sci Sports Exerc 39:117-125.

16 Lavoie JM, Leger, LA, Leone M, Provencher PJ (1985). A maximal multistage swim tet to determine the functional and maximal aerobic power of competitive swimmers. J Swimming Res 1:17-22.

17 Maglischo CW, Maglischo EW, Sharp RL, Zier DJ, Katz A (1984). Tethered and nontethered crawl swimming. In: J. Terauds, K. Barthels, E. Kreighbaum, R. Mann, J. Crakes, C.A. Del Mar (Eds) Proceedings of ISBS: Sports Biomechanics. Academic Publisher, 163-176.

18 Maglischo EW (1999). Nadando ainda mais rápido. São Paulo: Manole.
19 Marinho PC, Andries OJr (2001). Avaliação da força propulsora do nadador: validação e reprodutibilidade de uma metodologia específica. Rev. Bras. Ciência e Movimento, Edição especial (Supl), 79.

20 Mercier B, Granier P, Mercier J, Trouquet J, Préfaut CH (1993). Anaerobic and aerobic components during armcrank exercise in sprint and middle-distance swimmers. Eur J Appl Physiol 66:461-466.

21 Neufer PD, Costill DL, Fielding RA, Flynn MG, Kirwan JP (1987). Effect of reduced training on muscular strength and endurance in competitive swimmers. Med Sci Sports Exerc 19:486-490.

22 Papoti M, Martins LEB, Cunha SA, Freitas Jr PB, Gobatto, CA (2002). Effects of taper on swimming force and performance of swimmers after an experimental 10-wk training program. In: M. Coscolou, N. Geladas, V. Klissouras (Eds) Proceedings of the $7^{\text {th }}$ Annual Congres of the European College of Sport Science, 1:470.

23 Sharp RL, Troup JP, Costill DL (1982). Relationship between power and sprint freestyle swimming. Med Sci Sports Exerc 14:53-56.

24 Smith DJ, Norris SR, Hogg JM (2002). Performance Evalution of Swimmers: Scientific Tools. Sports Medicine 32:539-554.

25 Striet LL, Howlett RA, Heigenhauser GJF (2000). An enzymatic approach to lactate production in human skeletal muscle during exercise. Med Sci Sports Exerc 32:756-763.

26 Swaine IL, Zanker CL (1996). The Reproducibility of Cardiopulmonary Reponses to Exercise Using a Swim Bench. In J Sports Med 17:140-144.

27 Trappe S, Costill DL., Thomas, R (2001). Effect of swim taper on whole muscle and single muscle fiber contractile properties. Med Sci Sports Exerc 33:48-56.

28 Weinstein Y, Bediz C, Raffy D, Falk B (1998). Reliability of peak-lactate, heart rate, and plasma volume following the Wingate test. Med Sci Sports Exerc 30:1456-1460. 